DOI: 10.2478/linpo-2014-0006

\title{
New challenges in psycholinguistics: Interactivity and alignment in interpersonal communication
}

\author{
Maciej Karpiński \\ Institute of Linguistics, Adam Mickiewicz University in Poznań, \\ maciej.karpinski@amu.edu.pl
}

\begin{abstract}
Maciej Karpiński. New challenges in psycholinguistics:Interactivity and alignment in interpersonal communication. The Poznan Society for the Advancement of the Arts and Sciences. PL ISSN 0079-4740, ISBN 978-83-7654-384-0, pp. 97-106

In the present text, some recent changes in the perspective taken by psycholinguists in the study of language and communication are discussed. Their interests seem to gradually shift from the study of language processing as an isolated and independent phenomenon towards inclusion of more interactional factors being indispensable components of interpersonal communication and involved in the process of communicative alignment. Alignment is here understood as a complex phenomenon that goes beyond increasing similarity of mental representations and related communicative behaviour. It simultaneously occurs on many levels and in various modalities, including those traditionally excluded from language study. As a consequence, it implies not only more flexibility in the study of interpersonal communication but it also means a shift in the psycholinguistic methodology and probably also in the widely accepted picture of language and its limits.
\end{abstract}

Keywords: interactivity, alignment, entrainment, dialogue

\section{Introduction}

In recent years, the attention of many psycholinguists has gradually shifted from the individual minds of language users and strictly intra-personal aspects of language processing, towards a different but not necessarily contradictory perspective. Interactivity has come into play as an important factor that can hardly be ignored in the analysis of the mental mechanisms underlying language use. Language emerged from interaction and it has been bound to it during its evolution. Accordingly, to fully capture and understand communicative interactivity, one should take into account that some of its aspects are "embrained" - the neural structures responsible for communication are also interaction-oriented. Moreover, interactivity must also be somehow encoded in the language system itself. In his seminal book, Clark (1996) explicitly states that language should be investigated as a collaborative, joint activity, but even much earlier studies (e.g., Schegloff 1982) refer to discourse as a "an interactional achievement." 
A straightforward view of interactivity is that certain actions of one party result in certain actions of the other, which in turn may be noticed by the former and answered with another sequence of actions. Accordingly, one may be able to find some corresponding patterns of behaviour in the conversational partners, even though the notion of "correspondence" remains rather intuitive at this stage. The assumed correspondence may be based on synchrony (co-occurrence) or on any kind of similarity relationship between units of communicative behaviour. As a result, the activities of conversational parties can be viewed as co-ordinated (Garrod \& Anderson 1987).

Communicative interactivity is strictly bound to the co-ordination of communicative behaviour which can be observed on many levels, from simple physical activities to the dynamics of mental representations, as well as in many domains and modalities. One may assume that there is an inborn tendency to align which may direct humans towards increased interactivity. This tendency may be developed and strengthened due to immersion in society and culture. There are also some external factors, like music, that influence conversational partners and may result in some kind of alignment of their behaviour.

The present text is intended as a very compact review of various understandings and aspects of interactive alignment. Some issues related to its measurement and modelling are touched upon. Finally, an attempt is made to predict the directions of alignment research and its potential impact on psycholinguistics.

\section{Understanding and defining communicative alignment}

Although much of the innovative thought regarding alignment in communication is attributed to Pickering \& Garrod (2004), their seminal article was preceded and probably inspired by a sequence of earlier studies (e.g. Street 1984; Garrod \& Anderson 1987; Bernieri \& Rosenthal 1991), including the works of Giles (e.g. Giles et al. 1973; Giles \& Smith 1979). The importance of the notion of alignment seems to lie in the fact that it helps immensely to understand, model or explain a number of phenomena on the linguistic, paralinguistic and extralinguistic levels of conversation, while its measures make it possible to predict communication flow and its success or failure (Porzel et al. 2006; Reitter \& Moore 2007; Ramseyer \& Tschacher 2010). Its introduction shifts the focus of study away from the traditionally understood process of information transfer.

Alignment can be conceptualised as based on a particular type of priming that occurs as a continuous process in dialogue - two-way, mutual, but not necessarily symmetrical (cf. Krauss \& Pardo 2004). Both "simple" and structural priming can be involved here: similarity may occur and grow in terms of simple measures of specific phenomena as well as in terms of entire structures (e.g., for syntactic structures, see Branigan et al. 2000; Pietsch et al. 2012). Factors like social position, competence, or rhetorical skills may result in domination by one of the parties, and as a consequence in his/her greater influence on the other party's conversational behaviour. But even if one of the parties is dominant, the actual dominance is only possible if the behaviour of the other speaker is compatible and expresses his or her subordination.

Terms like alignment, convergence, co-occurrence, entrainment, synchrony, and probably some others, are defined from different viewpoints and perhaps with different purposes 
in mind (Levitan \& Hirschberg 2011), even though many of them are interchangeable in some contexts. Pickering and Garrod (2004) distinguish between alignment and co-ordination, the former related to the level of mental representations while the latter to the physical strata of behaviour. Accordingly, communicative alignment will be understood here as the process of mutually adjusting mental representations which are bound to various levels of communicative behaviour of conversation parties. Often, but not always, it will head towards increased similarity that can be captured as changes in some measurable values like pitch frequency or the range of hand movements, or of their synchronous co-variance. Since there have been some controversies regarding the conscious, sub- or unconscious nature of priming (see e.g. Krauss \& Pardo 2004) as well as automatic and strategic components of alignment (Kopp \& Bergmann 2013), no assumptions are made here regarding these dimensions. Nevertheless, alignment and co-ordination, as defined above, are bound together by numerous top-down and bottom-up processes, and these links open a way for their empirical exploration.

With what is still a tentative definition in hand, one is ready to pose more questions. Does communicative alignment reach its maximum at a certain stage of conversation? Is it maintained afterwards at a relatively stable level, or do its measures tend to fluctuate? What is the direction of alignment and how is it balanced? How can it be measured locally and globally? Is a stronger social position a prerequisite for being the "pivot" or the "reference point" of alignment, and to what degree and when can alignment be asymmetrical and still be understood as “interactive”? (Compare also Levitan's and Hirschberg's (2011) questions on the definition of entrainment.)

\section{The dynamics of context, coherence and communicative alignment}

The presence and role of context in language communication was acknowledged quite early in the history of contemporary linguistics. It was elaborated especially by those who dealt with various forms of texts and those who felt the importance of including social and interpersonal factors in the analysis of language communication. Various concepts and understandings of context have gradually emerged, ranging from those stemming from the textual perspective and the properties of text (de Beaugrande \& Dressler 1978), to those more anchored in psycholinguistics and psychology, stressing the aspect of individual perception and understanding (Givón 1995).

At least for a period of time, context of communication was viewed and analysed as a static set of conditions. But even its relatively early linguistic formalisations and accounts seem to have left some space for its dynamics (Hymes 1974) and incrementality (Sacks 1992) which was also bound to alignment by Purver \& Kempson (2004). Context may change as a result of its internal characteristics, but also evolve under the influence of human actors - the participants in the process of communication. Accordingly, context can also be viewed as interactive, as it may react to the actions of humans, while on the other hand it may influence them. Obviously, context may include not only objects but also animals or humans. Moreover, there is a mental component to it, as the attitudes, emotional states, prior 
knowledge and many similar factors can hardly be neglected, considering their potentially immense impact on the process of that communication.

Another important fact is that context may significantly contribute to coherence-related processes. Coherence, viewed as a mental process, and not directly as a property of printed text or a spoken utterance (Givón 1995), is achieved on the basis of a number of external cues that may come from surrounding objects, their properties or actions, but also from the mental content that is already present in the mind. Mills \& Gregoromichelaki (2010) point to the fact that Pickering's \& Garrod's (2004) model does not account for the sequentiality that is an inherent property of dialogue. This can obviously be regarded as a weaker point of this approach, although sequentiality is not indispensable for coherence. However, alignment itself is, even if not explicitly, analysed in most situations as a process in time, with events scattered along the time axis, forming sequences, and even the form of sequencing in the conversational partners may be analysed in terms of alignment processes.

\section{Modalities and channels, levels and areas of alignment}

One possible approach to ordering the streams of data between or among communicating people is to divide them into modalities, understood as related to senses, and into channels that can function quasi-independently within modalities (Karpiński 2009). For example, the ability of hearing corresponds to the auditory modality and embraces a number of possible channels. In language, the suprasegmental information can be conveyed quasi-independently from the segmental information even though segmentals and suprasegmentals influence each other mutually on various levels, including articulation. Of course, additional information comes from other sounds produced by the speaker (like knocking or whistling). In interpersonal communication, priority is given to auditory and visual modalities, although others may also introduce very important cues. It can also be hypothesised that language itself may play a pivotal role in the general process of alignment, due to its structural properties and the deep cultural grounding that influences ways of speaking. Giles et al. $(1973,2010)$ give the preliminaries to a theory of interpersonal accommodation through language.

Modalities are related to specific categories of mental representations and processing modes. Potentially, alignment can therefore be observed on various levels of representational hierarchies and linked to various categories of communicative behaviour, including gestures and speech. Accordingly, one can distinguish levels of representational alignment - not in the sense of its depth or strength, but rather in terms of more or less abstract categories of qualities for which it actually holds. For example, the area of phonological and articulatory alignment has been explored by Krauss \& Pardo (2004) and by Bard et al. (2000). A relatively large body of work has been devoted to issues of prosodic alignment, e.g. Street (1984), Kousidis et al. (2008), Edlund et al. (2009), Kousidis (2010), Kim et al. (2011), Levitan \& Hirschberg (2011), De Looze \& Rauzy (2011), Vaughan (2011), Truong \& Heylen (2012), and Włodarczak et al. (2012), Karpiński et al. (2014). Some of these works focus on a range of paralinguistic phenomena (such as the distribution of pauses). In the case of lexicon (Garrod \& Anderson 1987; Brennan \& Clark 1996) or syntax (Branigan et al. 2000), the issue of multiple and possibly parallel levels of the studied phenomenon raises a series of important questions. Increasingly similar vocabulary or syntactic structures in speakers 
during conversation may be, for example, regarded as a shallow, automatic phenomenon or else as involving deeper levels of cognitive processing (e.g. mental representations of situations, not only of objects or singular actions). A tentative answer is that "shallow", "physical" levels of interactions may be indispensable to provide data for high-level processing, and that these two levels are not only parallel but also closely interrelated.

High-level, semantic and conceptual alignment (Garrod \& Anderson 1987; Markman \& Makin 1998) can probably be viewed as resulting from both bottom-up processes (e.g. lower level alignment) as well as top-down processes, covering general tendencies in communicative behaviour, including the tendency to align itself. On an abstract and complex level, alignment has been traced in the domain of reference frames (Schober 1993; Watson et al. 2004) and situation models (Garrod \& Anderson 1987; Garrod \& Doherty 1994). Wagner et al. (2013) postulate that alignment may percolate across various levels of representation. This idea is crucial to their concept of Interactional Phonology but it results in serious consequences to many other levels of processing and language subsystems involved in the process of communication. Therefore, it is not surprising that alignment related to a certain modality or channel may be modified and mediated by others. For example, Murray-Smith et al. (2007) found that gait alignment occurred in people who were communicating using mobile phones and could not see each other during the conversation.

\section{How to capture and model alignment?}

Re-occurring words, particular intonation contours in both speakers, similar syntactic structures and other phenomena potentially related to alignment may also prove to be only accidental or to occur as a result of some other earlier or parallel processes, and thus not to stem directly from the tendency for ongoing co-ordination. Isolating these or filtering out irrelevant tendencies may require extensive use of statistics, and can be difficult when only sparse data are available.

Detection and measurement of alignment (or rather its physical manifestations) is far from trivial at any level of analysis. This is easily noticeable even when basic physical parameters of speech signal or gesticulation are considered. A direct, paired comparison of local measurements between speakers may prove difficult or not very productive. And even such a straightforward approach involves a lot of questions concerning the size of the time window for measurements, sampling, the time span at which some relationship may occur between the values measured for the two or more speakers, as well as the issues of normalisation. Furthermore, if alignment is to be treated as a process, the crucial issue would be to capture its dynamics - for example, the tendency of the measured values for two or more speakers to converge or diverge. Of course, the changes of values in the speech samples or gesture sequences under study may be more or less immediate. Finally, as mentioned, one may expect that at least in some areas, measures of alignment reach a certain maximum value, after which they do not increase further, but can only fluctuate.

In general, in the case of a separately treated, presumably (quasi-)independent parameters, such as pitch or intensity, one can take a number of different approaches: calculate a more or less complex similarity measure, look for co-variance of the values for the two or more speakers under study, track the similarity of their change patterns (which makes 
it possible to take account of possible delays or time shifts), or employ an approach based on time series or other statistics that can be applied to dynamic processes. When similarity measures are to be calculated for phenomena which, unlike pitch or intensity, are not directly expressible in numerical terms, or involve a number of dimensions, more advanced statistical methods, including artificial neural networks, may come into play.

Heldner et al. (2010) analyse entrainment locally in the domain of pitch, measuring pitch values in utterances and subsequent backchannels. A different approach is proposed by Kousidis et al. (2008; see also Vaughan 2011), who opt for the TAMA method based on a time-aligned moving average, which is essentially used to facilitate visual inspection of the alignment of, for example, prosodic parameters. Speech rate or pitch comparison may require testing various approaches to measurement and time window sizes (e.g. Buder \& Eriksson 1999; Boker et al. 2002). A limited time window may provide more information on local, immediate alignment-related phenomena, while a larger one may help to discover long-run or global tendencies (Karpiński et al. 2014).

The degree of similarity on the level of the acoustic signal can be measured not only by a more or less direct comparison of phonetic-acoustic parameters (as in works cited above) but also by means of perception tasks (e.g. Pardo 2006). In a number of studies, researchers move forward from hypotheses through observation to experimental exploration and modelling. Mehler et al. (2010; 2011) propose to operationalise and model the notion of interpersonal alignment in dialogue in terms of structural similarity on the lexical level using two-layer time-aligned network series (a time-adjusted graph model).

In any case, higher similarity measures in the final stage of a dialogue than at its beginning may prove to be accidental or caused by some external factors, and not by the process of alignment. For example, the acoustic environment may turn noisy, causing both the speakers to speak at higher energy levels. Therefore, it may prove necessary to track the entire process of conversation, to distinguish its stages, and to search for alignment within and between or among the stages, taking into account both immediate and more time-stretched phenomena.

Another potential issue is that while the studies dealing with physical parameters rather than linguistic units may provide more freedom and probably easier quantitative analyses, their results may be more difficult to interpret and generalise in linguistic terms (if such a need arises).

\section{How far can interactivity and alignment go?}

One aspect of the question posed as the title of this section is that of the actual weight of alignment-related phenomena in models of psycholinguistic processes. Even if one assumes that alignment is the major force that shapes the process of communication, there are a number of other potentially influential factors. Integration of context and alignment modelling may be worth consideration.

Another aspect which requires serious consideration is the scope and domain of alignment. Passive and active components of the physical environment can also be viewed as playing their roles in a holistically captured process of alignment. Some of them can hardly be modified by the human participants of conversation (e.g. certain physical settings like the 
shape of a given room), while others may change as a result of a physical action (a door may be opened). Even if this kind of "co-ordination" between humans and their physical environment is not always as interactive as that which occurs directly between or among human beings themselves, a generalised model does not have to assume that all of the components are active and interact to the same degree. The same applies to the mental component of context, which both influences and is influenced by the flow of communication, while its various parts may be flexible to different degrees.

Alignment can be viewed as a not fully conscious, "resource free" process, but in a wide range of situations it can be also manipulated, consciously adjusted or even "acted." On the other hand, speakers may more or less consciously break this process by, for example, "desynchronising" their dialogue contribution, suddenly changing the repertoire of vocabulary and syntactic structures or rapidly switching their tone of voice. Such changes may be fully purposeful and deliberate. When one of the dialogue parties is extremely distressed, speaking in a high voice at a high rate, the other may use lower voice and deliberately slowed-down tempo in order to calm down the conversation and compensate for the extreme emotions of his/her conversational partner. But one can also postulate the existence of mechanisms that automatically ensure that a certain level of similarity in behaviour (as well as on the level of mental representations) is maintained as optimum or just not exceeded.

Weiß et al. (2009) show that local alignment on the lexical level may gradually "drift apart", and introduce the notion of anti-alignment. One can hypothesise that if the initial level of convergence between speakers (on various levels) is extremely high, they may intuitively or consciously take steps to protect their identity and stress the differences in the way they speak, up to a point at which they achieve a degree of safety. Reiter \& Moore (2007) point to another issue that raises further doubts related to the process of alignment, arguing that repetitions in dialogue result from adaptation problems and not necessarily from short-term entrainment. Alignment should be regarded as a multi-dimensional and multidirectional process. As has been shown above, it can be observed in multiple dimensions of communication. Viewing it as a process directed solely towards increased convergence, similarity or synchrony is probably a simplification. It should rather be modelled as a tendency towards a balance between various "aligning forces" and a number of "disaligning" factors (including speakers' individual features and the abovementioned intentional lack of alignment).

Another aspect of the initial question is the potential range of impact of the alignmentfocused approach on various areas of psycholinguistics and models of psycholinguistic processes. In the Interactive Alignment Model of dialogue (Pickering \& Garrod 2004) it is assumed that the alignment of situation models is caused by automatic and resource-free priming-based mechanisms at all levels of linguistic processing. This ambitious attempt shows how a convincing model of language communication can be built around the notion of alignment. On the other hand, after years, it still calls for more empirical support and more "instantiations" grounded in various dimensions of interpersonal communication.

Interactivity has been a feature of numerous psycholinguistic models for quite a long time, preparing, in a way, for the introduction of the concept of alignment as a pivotal component of interaction. One of the first attempts to incorporate interpersonal alignment-related mechanisms into not only models of communication but also directly into language 
processing is the interactional phonology proposed by Wagner et al. (2013). Another direction that will have to be taken is to investigate the neural underpinnings of alignment, and some steps in this direction have already been taken (e.g. Menetti et al. 2012).

Although the concepts of interactivity and alignment are being further elaborated and their understanding may still evolve, they have already played an important role in the psycholinguistic exploration of the flow of conversation. Their introduction may have further consequences in various subfields of psycholinguistics. It should make psycholinguists widen the scope of their studies to include all types of communicatively relevant behaviour that may be involved in the process of interactive alignment and, as a consequence, influence and reshape current models of speech production, perception and understanding.

\section{References}

Bard, Ellen G. \& Anderson, Anne H. \& Sotillo, Catherine \& Aylett, Mathew \& Doherty-Sneddon, Gwyneth $\&$ Newlands, Allison. 2000. Controlling the intelligibility of referring expressions in dialogue. Journal of Memory and Language 42. 1-22.

Bergmann, Kirsten \& Kopp, Stefan. 2012. Gestural alignment in natural dialogue. In Cooper, R.P. \& Peebles, D. $\&$ Miyake, N. (eds.) Proceedings of the 34th Annual Conference of the Cognitive Science Society (CogSci 2012), 1326-1331. Austin, TX: Cognitive Science Society.

Bernieri, Frank J. \& Rosenthal, Robert. 1991. Interpersonal coordination: Behaviour matching and interactional synchrony. In Feldman, Robert \& Rimé, Bertrand (eds.), Fundamentals of non-verbal behaviour, 401-432. New York: Cambridge University Press.

Boker, Steven M. \& Xu, Minquan \& Rotondo, Jennifer L. \& King, Kadija 2002. Windowed cross-correlation and peak picking for the analysis of variability in the association between behavioural time series, Psychological Methods 7. 338-355.

Branigan, Holly P. \& Pickering, Martin P. \& Cleland, Alexandra A. 2000. Syntactic co-ordination in dialogue. Cognition 75. 13-25.

Brennan, Susan E. \& Clark, Herbert H. 1996. Conceptual pacts and lexical choice in conversation. Journal of Experimental Psychology: Learning, Memory, and Cognition 22. 1482-1493.

Buder, Eugene H. \& Eriksson, Anders. 1999. Time-series analysis of conversational prosody for the identification of rhythmic units. In Proceedings of the 14th International Congress of Phonetic Sciences, San Francisco, 1-7 August 1999. 1071-1074.

de Beaugrande, Robert \& Dressler, Wolfgang. 1981. Introduction to Text Linguistics. London: Longman.

Clark, Herbert H. 1996. Using language. Cambridge: Cambridge University Press.

Edlund, Jens \& Heldner, Martin \& Hirschberg, Julia. 2009. Pause and gap length in face-to-face interaction, Proceedings of Interspeech 2009, Brno. 2779-2782.

Garrod, Simon C. \& Anderson, Anthony. 1987. Saying what you mean in dialogue: A study in conceptual and semantic coordination. Cognition 27. 181-218.

Garrod, Simon C. \& Doherty, Gwyneth. 1994. Conversation, co-ordination and convention: and empirical investigation of how groups establish linguistic conventions. Cognition 53. 181-215.

Garrod, Simon C. \& Pickering, Martin J. 2004. Why is conversation so easy? Trends in Cognitive Sciences 8. $8-11$.

Giles, Howard \& Smith, Philip M. 1979. Accommodation theory: Optimal levels of convergence. In Giles, Howard \& St. Clair, Robert N. (eds.), Language and social psychology, 45-65. Baltimore: University Park Press.

Giles, Howard \& Taylor, Donald M. \& Bourhis, Richard \& Rosenthal, Dorrin. 2010. Towards a theory of interpersonal accommodation through language: Some Canadian data. Language in Society 2. 177-192.

Giles, Howard \& Taylor, Donald M. \& Bourhis, Richard Y. 1973. Towards a theory of interpersonal accommodation through language: Some Canadian data. Language in Society 2. 177-192.

Givón, Talmy. 1995. Coherence in text vs. coherence in mind. In Gernsbacher, Morton Ann \& Givón, Talmy (eds.), Coherence in spontaneous text, 95-115. Amsterdam: John Benjamins Publishing Company. 
Heldner, Martin \& Edlund, Jens \& Hirschberg, Julia. 2010. Pitch similarity in the vicinity of backchannels, In Proceedings of Interspeech 2010, Makuhari 3054-3057.

Hymes, Dell. 1974. Foundations of sociolinguistics: An ethnographic approach. Philadelphia: University of Pennsylvania.

Karpiński, Maciej. 2009. From speech and gestures to dialogue acts. In Esposito, Anna \& Hussain, Amir \& Marinaro, Mario \& Martone, Raffaele (eds.), Multimodal signals: Cognitive and algorithmic issues, 164 169. Heidelberg: Springer Verlag.

Karpiński, Maciej \& Klessa, Katarzyna \& Czoska, Agnieszka. 2014. Local and global convergence in the temporal domain in Polish task-oriented dialogue. In Campbell, N. \& Gibbon, Dafydd \& Hirst, Daniel (eds.), Proceedings of Speech Prosody 7 Conference. Dublin, 743-747.

Kim, Midam \& Horton, Sid \& Bradlow, Ann R. 2011. Phonetic convergence in spontaneous conversations as a function of interlocutor language distance. Laboratory Phonology 2. 125-156.

Kopp, Stefan \& Bergmann, Kirsten 2013. Automatic and strategic alignment of co-verbal gestures in dialogue. In Wachsmuth, Ipke \& de Ruiter, Jan \& Jaecks, Petra \& Kopp, Stefan (eds.) Alignment in Communication, 87-107. Amsterdam: John Benjamins Publishing Company.

Kousidis, Spyros. 2010. A study of accommodation of prosodic and temporal features in spoken dialogues in view of speech technology applications. Dublin: Dublin Institute of Technology. [Doctoral dissertation.]

Kousidis, Spyros \& Dorran, David \& Wang, Yi \& Vaughan, Brian \& Cullen, Charlie \& Campbell, Dermont \& McDonnell, Ciaran \& Coyle, Eugene 2008. Towards measuring continuous acoustic feature convergence in unconstrained spoken dialogues. In Proceedings of Interspeech 2008, Brisbane, 1692-1695.

Krauss, Robert M. \& Pardo, Jennifer. S. 2004. Is alignment always the result of automatic priming? Behavioral and Brain Sciences 27(02). 203-204.

Levitan, Rivka \& Hirschberg, Julia. 2011. Measuring acoustic-prosodic entrainment with respect to multiple levels and dimensions, Proceedings of Interspeech 2011, Florence, Italy, 3081-3084.

Looze, de, Céline \& Rauzy, Stephane. 2011. Measuring speakers' similarity in speech by means of prosodic cues: methods and potential. Proceedings of Interspeech, Florence, Italy, 1393-1396.

Markman, Arthur B. \& Makin, Valerie S. 1998. Referential communication and category acquisition. Journal of Experimental Psychology 127. 331-354.

Mehler, Alexander \& Lücking, Andy \& Menke, Peter. 2011. Modelling Lexical Alignment in spontaneous Direction Dialogue Data by Means of a Lexicon Network Model. In Proceedings of 12th International Conference on Intelligent Text Processing and Computational Linguistics (CICLing), February 20-26, Tokyo. Berlin: Springer.

Mehler, Alexander \& Lücking, Andy \& Weiß, Petra. 2010. A network model of interpersonal alignment in dialog. Entropy 12. 1440-1483.

Menenti, Laura \& Pickering, Martin J. \& Garrod, Simon C. 2012. Towards a neural basis of interactive alignment in conversation. Frontiers in Human Neuroscience 6, art. 185. 1-9. (10.3389/fnhum.2012.00185)

Mills, Gregory \& Gregoromichelaki, Eleni. 2010. Establishing coherence in dialogue: Sequentiality, intentions and negotiation. In Łupkowski, Paweł \& Purver, Matthew (eds.), Proceedings of SemDial (PozDial), 14th Workshop on Semantics and Pragmatics of Dialogue. Poznań: Polish Society for Cognitive Science. 17-24.

Murray-Smith, Roderick \& Ramsay, Andrew \& Garrod, Simon \& Jackson, Melissa \& Musizza, Bojan. 2007. Gait alignment in mobile phone conversations. In Proceedings of the 9th International Conferences on HumanComputer Interaction with Mobile Devices and Services (Singapore, September 09 - 12, 2007). MobileHCI '07, vol. 309. ACM, New York, NY, 214-221.

Pardo, Jennifer. 2006. On phonetic convergence during conversational interaction. Journal of the Acoustical Society of America 119. 2382-2393.

Pickering, Martin J. \& Garrod, Simon C. 2004. Toward a mechanistic psychology of dialogue. Behavioural and Brain Sciences 27. 169-226.

Pickering, Martin J. \& Garrod, Simon C. 2006. Alignment as the basis for successful communication. Research on Language and Computation 4(2-3). 203-228.

Pietsch, Christian \& Buch, Armin \& Kopp, Stefan \& de Ruiter, Jan. 2012. Measuring syntactic priming in dialogue Corpora. In Stolterfoht, Britta \& Featherston, Sam (eds.), Empirical approaches to linguistic theory: Studies of meaning and structure, 29-41. The Hague: Mouton de Gruyter.

Porzel, Robert \& Scheffler, Annika \& Malaka, Rainer. 2006. How entrainment increases dialogical efficiency. In Proceedings of Workshop on Effective Multimodal Dialogue Interfaces, Sydney 2006. 
Purver, Matthew \& Kempson, Ruth. 2004. Incremental parsing, or incremental grammar? In Proceedings of the ACL Workshop on Incremental Parsing, Barcelona, July 2004, 74-81.

Ramseyer, Fabian \& Tschacher, Wolfgang. 2010. Nonverbal synchrony or random coincidence? How to tell the difference. In Esposito, Anna \& Campbell, Nick \& Vogel, Carl \& Hussain, Amir \& Nijholt, Amir (eds.), Development of multimodal interfaces: Active listening and synchrony, 182-196. Heidelberg: Springer Verlag.

Reitter, David \& Moore, Johanna D. 2007. Predicting success in dialogue. In Proceedings of the 45th Annual Meeting of the Association of Computational Linguistics, Prague, 808-815.

Sacks, Harvey. 1992. Lectures on Conversation. Oxford: Blackwell.

Schegloff, Emmanuel A. 1982. Discourse as an interactional achievement: Some uses of 'uh huh' and other things that come between sentences. In Tannen, Deborah (ed.) Analyzing Discourse: Text and Talk, 71-93. Georgetown: Georgetown University Press.

Schober, Michael F. 1993. Spatial perspective-taking in conversation. Cognition 47. 1-24.

Street, Richard L. 1984. Speech convergence and evaluation in fact-finding interviews. Human Communication Research 11(2). 139-169.

Street, Richard L. \& Brady, Robert M. \& Putman, William B. 1983. The influence of speech rate stereotypes and rate similarity or listeners' evaluations of speakers. Journal of Language and Social Psychology 2(1). 37-56.

Truong, Khiet P. \& Heylen, Dirk. 2012. Measuring prosodic alignment in cooperative task-based conversations. In Proceedings of Interspeech 2012, Portland, OR, USA, 1085-1088.

Vaughan, Brian. 2011. Prosodic synchrony in co-operative task-based dialogues: A measure of agreement and disagreement. In Proceedings of Interspeech 2011, 1865-1867.

Wagner, Petra \& Malisz, Zofia \& Inden, Benjamin \& Wachsmuth, Ipke. 2013. Interaction phonology - a temporal co-ordination component enabling representational alignment within a model of communication. In Wachsmuth, Ipke \& de Ruiter, Jan \& Jaecks, Petra \& Kopp, Stefan (eds.) Alignment in communication: Towards a new theory of communication. Advances in interaction studies, 109-132. Amsterdam: Benjamins.

Watson, Mathew E. \& Pickering, Martin J. \& Branigan, Holly P. 2004. Alignment of reference frames in dialogue. In Proceedings of the 26th Annual Conference of the Cognitive Science Society, 2353-2358. Chicago: Erlbaum Associates.

Weiß, P. \& Pustylnikov, Olga \& Mehler, Alexander \& Hellmann, Sara. 2009. Patterns of alignment in dialogue: Conversational partners do not always stay aligned on common object names. In Conference on Embodied and Situated Language Processing (ESLP 2009). Rotterdam, 16.

Włodarczak, Marcin \& Šimko, Juraj \& Wagner, Petra. 2012. Syllable boundary effect: Temporal entrainment in overlapped speech. In Ma, Qiuwu \& Ding, Hongwei \& Hirst, Daniel (eds.), Proceedings of Speech Prosody 2012, Shanghai, 611-614. 